\title{
Cytosolic DNA sensing through cGAS and STING is inactivated by gene mutations in pangolins
}

\author{
Heinz Fischer ${ }^{1} \cdot$ Erwin Tschachler $^{2} \cdot$ Leopold Eckhart $^{2}{ }^{2}$
}

Published online: 12 June 2020

(c) The Author(s) 2020

\begin{abstract}
The release of DNA into the cytoplasm upon damage to the nucleus or during viral infection triggers an interferon-mediated defense response, inflammation and cell death. In human cells cytoplasmic DNA is sensed by cyclic GMP-AMP Synthase (cGAS) and Absent In Melanoma 2 (AIM2). Here, we report the identification of a "natural knockout" model of cGAS. Comparative genomics of phylogenetically diverse mammalian species showed that cGAS and its interaction partner Stimulator of Interferon Genes (STING) have been inactivated by mutations in the Malayan pangolin whereas other mammals retained intact copies of these genes. The coding sequences of CGAS and STING1 are also disrupted by premature stop codons and frame-shift mutations in Chinese and tree pangolins, suggesting that expression of these genes was lost in a common ancestor of all pangolins that lived more than 20 million years ago. AIM2 is retained in a functional form in pangolins whereas it is inactivated by mutations in carnivorans, the phylogenetic sister group of pangolins. The deficiency of cGAS and STING points to the existence of alternative mechanisms of controlling cytoplasmic DNA-associated cell damage and viral infections in pangolins.
\end{abstract}

Keywords DNA sensor $\cdot$ Innate immunity $\cdot$ Inflammation $\cdot$ Pangolin $\cdot$ Zoonosis $\cdot$ Gene loss

\section{Introduction}

The presence of DNA in the cytoplasm is a sign of infection with DNA viruses or damage to the nucleus or mitochondria in which endogenous DNA is normally contained [1, 2]. During mitosis the nuclear membrane disintegrates but DNA is compacted and tightly covered by histones so that it is not directly accessible to cytoplasmic proteins [3]. Cellular response systems have evolved to detect cytoplasmic DNA and to initiate processes aimed at the re-establishment of homeostasis at the tissue level. These responses include the production of type I and type III interferons, interleukin

Electronic supplementary material The online version of this article (https://doi.org/10.1007/s10495-020-01614-4) contains supplementary material, which is available to authorized users.

Leopold Eckhart

leopold.eckhart@meduniwien.ac.at

1 Division of Cell and Developmental Biology, Center for Anatomy and Cell Biology, Medical University of Vienna, Vienna, Austria

2 Department of Dermatology, Medical University of Vienna, Vienna, Austria
(IL)-1 and 18 which transmit a danger signal to neighboring cells and activate the immune system. At the cellular level, mislocalized DNA can trigger senescence and programmed cell death [4-6]. Cell death is particularly important in host defense against DNA viruses, such as vaccinia virus, enterovirus A71, and herpes viruses, and bacteria, such as Mycobacterium tuberculosis variant bovis, Listeria monocytogenes, Legionella pneumophila, and Francisella tularenis, but also in sterile inflammation and cancer $[5,6]$.

Cytoplasmic DNA is sensed by Absent in melanoma 2 (AIM2) and cyclic GMP-AMP synthase (cGAS). AIM2 activates inflammasome-dependent IL- $1 \beta$ and a pro-inflammatory mode of cell death known as pyroptosis [7-10]. cGAS binds DNA and catalyzes the production of 2' 3 'cGAMP which is the ligand of Stimulator of interferon genes (STING) [11-13]. Subsequently, STING translocates to the Golgi and undergoes phosphorylation by TANK-binding kinase 1 (TBK1). Interferon regulatory factor (IRF) 3 is recruited and phosphorylated and the expression of interferon genes is induced $[4,14]$. By an as-yet unclear mechanism, also NF- $\mathrm{kB}$ is activated by STING, leading to the expression of inflammatory cytokines such as tumor necrosis factor (TNF). Via TNF and other pathways, activation of 
the cGAS-STING pathway triggers various types of programmed cell death including, necroptosis, apoptosis, and lysosomal cell death $[5,6]$.

Cytoplasmic DNA sensors have originated early in evolution [15] and homologs of AIM2 and cGAS were present in the first mammals [16-18]. Surprisingly, AIM2 is not conserved in cattle, dog, bats, and several other mammals [18-20], suggesting inter-species differences in the response to cytoplasmic DNA and dispensability of AIM2-mediated DNA-sensing for some species.

Pangolins are specialized insectivorous mammals that are phylogenetically most closely related to carnivorans. The body of pangolins is covered by keratinous scales which serve as a protective armor. Few comparative studies of the mammalian immune defense have included pangolins but the interest in pangolins has increased recently due their possible role as intermediate hosts for the pandemic severe acute respiratory syndrome coronavirus 2 (SARS-CoV-2) [21-23]. We have recently reported that interferon-induced with helicase C domain 1 (IFIH1)/MDA5, a sensor of intracellular double-stranded RNA, and Z-DNA-binding protein (ZBP1), which senses both Z-RNA and Z-DNA, have been lost during the evolution of pangolins [24]. Moreover, tolllike receptor (TLR) 5, the receptor of bacterial flagellin [25] and interferon- $\varepsilon$, a type I interferon that is expressed in epithelia of other mammals, have been lost in pangolins [26].

Here we investigated whether the genes controlling cytoplasmic DNA-sensing are conserved in pangolins and found that pseudogenization of critical genes has inactivated the cGAS-STING pathway that is implicated in the innate defense against DNA viruses and cytoplasmic DNA-stimulated cell death.

\section{Materials and methods}

Genes were identified in the genome sequences of the Malayan pangolin (Manis javanica), Assembly: ManJav1.0 (GCA_001685135.1), submitted by the International Pangolin Research Consortium (Choo et al. [26]); Chinese pangolin (Manis pentadactyla), Assembly: M_pentadactyla-1.1.1 (GCA_000738955.1), submitted by Washington University; Tree pangolin (Manis tricuspis), Assembly: ManTri_v1_ BIUU (GCA_004765945.1), submitted by Broad Institute. At the time of this study (April 2020) GenBank gene annotations were available for M. javanica (NCBI Manis javanica Annotation Release 100) but not for the other species of pangolins. Other nucleotide sequences were downloaded from GenBank and accession numbers are indicated in the text.

The Basic Local Alignment Search Tool (BLAST) was used to identify regions of sequence similarity [27]. Nucleotide sequence were translated into amino acid sequences using the Translate tool at the Expasy website of the Swiss
Institute of Bioinformatics (https://web.expasy.org/translate/). Sequence alignments were made with Multalin [28]. The Timetree website was used as a reference for phylogenetic relationships and divergence times (www.timetree.org) [29].

\section{Results}

\section{CGAS is inactivated by gene mutations in pangolins}

Comparative genomics of mammals showed conservation of the $C G A S$ gene locus in species from all major clades investigated except for the Malayan pangolin (Fig. 1a; Suppl. Table S1). The genes DDX43 and MTO1 which flank CGAS in mammalian genomes, are conserved in the pangolin but no gene is annotated between them. A targeted search for $C G A S$-like sequences revealed that remnants of 3 exons of CGAS are located between DDX43 and MTO1 in the pangolin (Fig. 1b). The coding sequence in each of these exon remnants was disrupted by premature in-frame stop codons and frame-shift mutations that prevent the translation into a functional protein. Analysis of the as-yet-unassembled whole genome shotgun sequences of two further pangolin species, i.e. Chinese pangolin and tree pangolin, showed also presence of disruptive mutations in CGAS. At least one these mutations, a premature stop codon in CGAS exon 3 (Fig. 1c) was conserved in all three species of pangolins, suggesting that this mutation was inherited from a common ancestor.

\section{STING is inactivated by gene mutations in pangolins}

Comparative genomics of mammals showed conservation of the STING1 (the gene encoding STING) locus in all species investigated including the Malayan pangolin (Fig. 2a). However, the exons of pangolin STING1 contained premature inframe stop codons and frame-shift mutations that disrupted the coding sequence at multiple sites (Fig. 2b-d). Analysis of whole genome shotgun sequences of Chinese and tree pangolins showed conserved disruptive mutations in exons 1 (Fig. 2c) and 4 (Fig. 2d) of STING1, besides further mutations that were present in either 1 or 2 of the 3 species of pangolins investigated.

In contrast to CGAS and STING1, AIM2 is intact in the Malayan pangolin (Suppl. Table S1) but mutated in various mammals [18-20]. IFI16, which belongs to the same protein family as AIM2 but reportedly controls DNA sensing through interactions with STING [30-32], was identified at the gene level in the cat and dog (Suppl. Table S1) but not in the Malayan pangolin (Suppl. Table S1). Genes encoding the endosomal DNA sensor TLR9 and genes controlling signaling downstream of DNA sensors such as TBK1, MYD88, $A S C / P Y C A R D$, and CASPI are intact in the Malayan pangolin (M. javanica) (Suppl. Table S1). 
a

Pangolin

DDX43 CGAS MT01

Cat

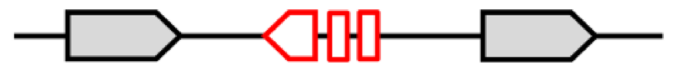

Human
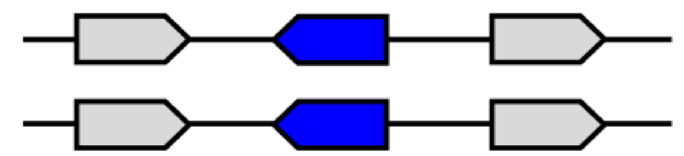

b

\section{Stop / frame-shift mutations}

Pangolin

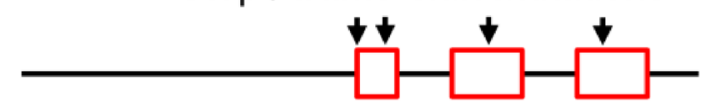

Cat

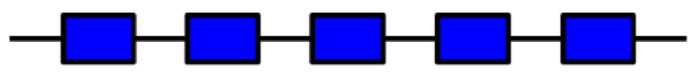

Human

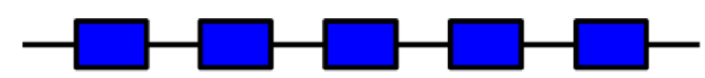

Coding exon:

1

2

3

4

5

C

Malayan pangolin Chinese pangolin

Tree pangolin

Cat

Human

\section{CGAS exon 3}

stop
TGGCTTGGAACAAAAGTTAAGAGCAGTCTAAGATGACAGCCATGTCACCTGGTACCCGAG
TGGCTTGGAACAAAAGTTAAGAGCAGTCTAAGATGACAGCCATGTCACCTGGTACCCGAG
TGGCTTGGAACAAAAGTTAAGAGCTGTCTACGATGACAGCCATGTCACGTGGTACCCAAG
TGGCTTGGAACAAAAGTTAGAAGCAGTCTAAGACAGCAGCCATTTTACCTGGTCCCCAAG
TGGCTTTCAGCAAAAGTTAGGAAGCAACTACGACTAAAGCCATTTTACCTTGTACCCAAG
Fig. 1 CGAS is a pseudogene in pangolins. a Gene locus of CGAS in the pangolin (M. javanica), cat, and human. Genes are represented by arrows pointing in the direction of transcription. A fragmented arrow indicates disruption of the coding sequence. b Exon-intron organization of CGAS in the pangolin (M. javanica), cat, and human. Exons containing coding sequence are indicated by boxes. Note that homologs of the first two exons could not be identified in the pangolin. Mutations leading to premature in-frame stop codons or reading frame-shifts in the pangolin gene are indicated. c Nucleotide sequence alignment of homologous segments of exon 3 of CGAS of three species of pangolins, cat and human. The coding sequence of human $C G A S$ was translated and the amino acid sequence is shown

\section{The evolutionary loss of cGAS and STING occurred after the divergence of pangolins from carnivorans whereas the latter lost AIM2}

The next relatives to pangolins are the carnivorans, including cat, dog, bears, pinnipeds and others. All carnivorans investigated have intact $C G A S$ and STINGI genes (Suppl. Table S1) but no functional AIM2 gene (Fig. 3). The below the nucleotide sequences. In-frame stop codons are highlighted by red shading. Nucleotides conserved in all species are indicated by blue fonts. Nucleotide sequence accession numbers (GenBank): Human (NC_000006.12, nucl. 73440237-73440296, compl.), cat (NC_018727.3, nucl. 69116688-69116747, compl.), Malayan pangolin (Manis javanica) (NW_016557438.1, nucl. 39779-39838, compl.), Chinese pangolin (Manis pentadactyla) (JPTV01097288.1, nucl. 22408-22467, compl.), tree pangolin (Manis tricuspis) (SOZM010013487.1, nucl. 43424-43483, compl.). Abbreviations: compl., complementary; nucl., nucleotide numbers (Color figure online)

species distribution of intact genes suggested that $C G A S$ and STING1 were inactivated by mutations in the lineage leading to pangolins and AIM2 was lost early in the evolution of carnivorans as well as in the evolution of other mammals such as cattle (Fig. 3a). Thus, each of the two main pathways of cytoplasmic DNA sensing is inactivated in at least one clade of mammals. 
a

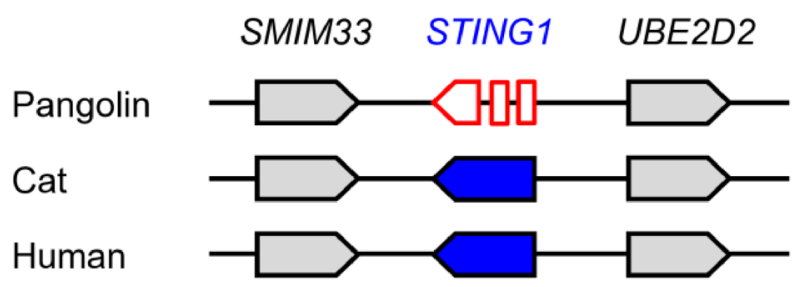

b

\section{C \\ Malayan pangolin Chinese pangolin Tree pangolin}

Cat

Human

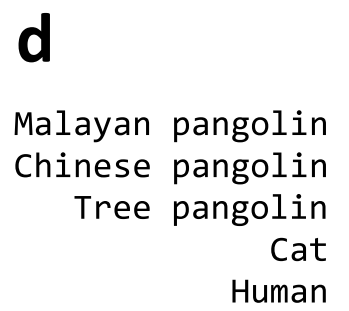

STING1 exon 1

$\begin{array}{cc}\text { frame-shift } & \text { stop } \\ \text { ATGCCTCACTCCAGC-TGCACCCACCCGTCCCGTGGCCCTCGGGTCGCTGAGTCCAGAAG } & \\ \text { ACGCCTCACTCCAGC-TGTACCCACCCGTCCCATGGCCCTCGGGTCGCTGAGTCCAGAAG } & \text { ACGCCTCACTCTAGCCTGCGCCCATCCATCCCATGGCCCACGGGTCACTGAGTCCAGAAG }\end{array}$ ATGCCCCGCACTGGCCTGCACCCATCCATCCCGAGGCCCAGGGGCATGGGGGCCCAGAAG ATGCCCCACTCCAGCCTGCATCCATCCATCCCGTGTCCCAGGGGTCACGGGGCCCAGAAG

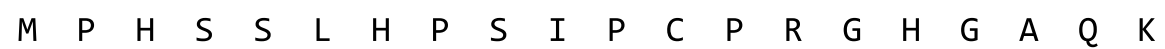

\section{STING1 exon 4}

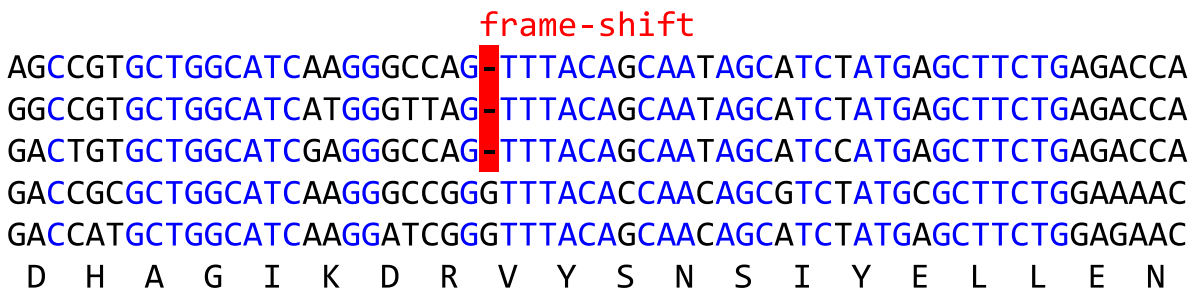

Fig. 2 STING1 is a pseudogene in pangolins. a Gene locus of STING1 in the pangolin (M. javanica), cat, and human. Genes are represented by arrows pointing in the direction of transcription. A fragmented arrow indicates disruption of the coding sequence. b Exon-intron organization of STING1 in the pangolin (M. javanica), cat, and human. Exons containing coding sequence are indicated by boxes. Mutations leading to premature in-frame stop codons or reading frame-shifts in the pangolin gene are indicated. c, d Nucleotide sequence alignment of homologous segments of exon 1 (c) and exon 4 (d) of STING1 of three species of pangolins, cat and human. The coding sequence of human STING1 was translated and the amino acid sequence is shown below the nucleotide sequences. Frame- shift mutations and in-frame stop codons in the pangolin genes are highlighted by red shading. Nucleotides conserved in all species are indicated by blue fonts. Nucleotide sequence accession numbers (GenBank): Human (NC_000005.10, nucl. 139481645-139481704, compl., 139478279-139478338, compl.), cat (NC_018723.3, nucl. 117883758-117883817, compl., 117881535-117881594, compl.), Malayan pangolin (Manis javanica) (NW_016540155.1, nucl. 4518245240, 47071-47129), Chinese pangolin (Manis pentadactyla) (JPTV01042841.1, nucl. 8735-8793, compl., 6847-6905, compl.), tree pangolin (Manis tricuspis) (SOZM010002209.1, nucl. 9193291991, compl., 90035-90093, compl.). Abbreviations: compl., complementary; nucl., nucleotide numbers (Color figure online) 
a

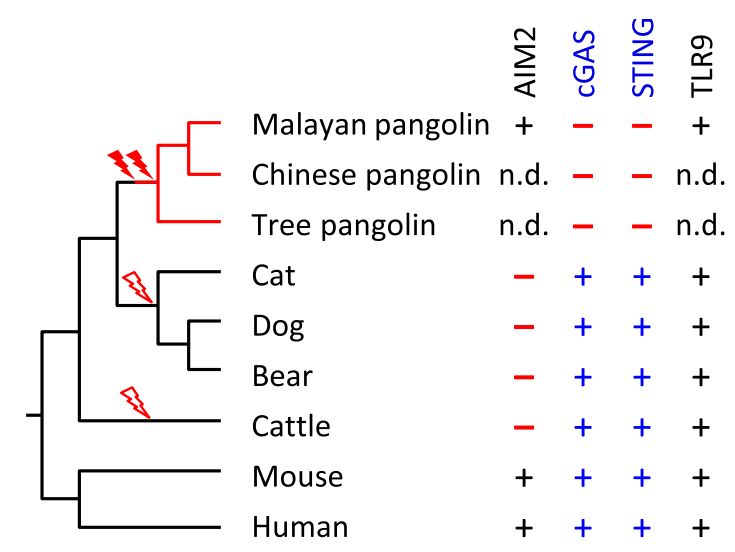

b

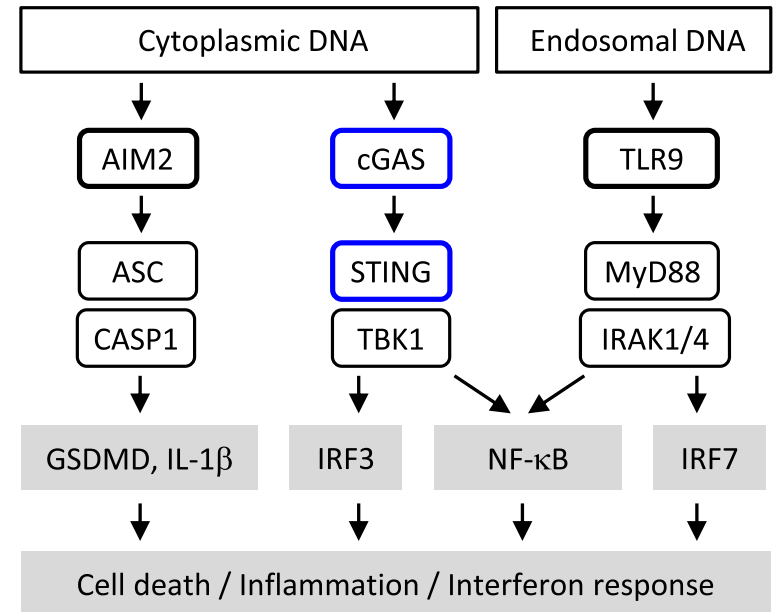

Fig. 3 Evolution of cytoplasmic DNA signaling in pangolins and other mammals. a Phylogenetic tree of mammals and comparison of presence $(+)$ or absence $(-)$ of DNA sensor genes. Evolutionary gene loss (indicated by lightning bolt symbols) was inferred from the species distribution of the genes. Species: Malayan pangolin (Manis javanica), Chinese pangolin (Manis pentadactyla), tree pangolin (Manis tricuspis), cat (Felis catus), dog (Canis lupus familiaris), bear (Ursus arctos horribilis), cattle (Bos taurus), mouse (Mus musculus), human (Homo sapiens). b Schematic overview of innate immune sensors of cytoplasmic and endosomal DNA and signaling in mammals. Only DNA sensors investigated in this study and a subset of the signaling proteins are shown. Abbreviations: AIM2, absent in melanoma 2; ASC, Apoptosis-associated speck like protein containing a caspase recruitment domain; CASP1, caspase-1; CGAS, cyclic GMP-AMP synthase; GSDMD, gasdermin D; IL- $1 \beta$, interleukin-1 $\beta$; IRAK, interleukin-1 receptor-associated kinase; IRF, interferon regulatory factors; NF- $\mathrm{BB}$, nuclear factor kappa-light-chain-enhancer of activated B cells; MYD88, myeloid differentiation primary response 88; STING1, stimulator of interferon genes; TBK1, TANK-binding kinase 1; TLR9, toll-like receptor 9; n.d., not determined

\section{Discussion}

To the best of our knowledge, pangolins are the first mammals that are reported to lack the cGAS-STING pathway.
Strikingly, both genes that are specifically active in this pathway, i.e. CGAS and STING1, are pseudogenized in pangolins and the results of our comparative analysis of phylogenetically diverse pangolin species suggest that the inactivating mutations occurred more than 20 million years ago. We can conclude that the cGAS-STING pathway has been dispensable for the survival of pangolins and it is even conceivable that the loss of the cGAS-STING-dependent response to cytoplasmic DNA provided an evolutionary advantage.

We have recently reported the loss of the IFIH1/MDA5dependent response to double-stranded RNA in pangolins [24], leading us to put forward the hypothesis that a diminished innate immune response to certain RNA viruses, including coronaviruses, may avoid overshooting host defense reactions and loss of IFIH1 may have allowed for the evolution of tolerance as a strategy to survive viral infections. The results of the present study show that also a major pathway of sensing DNA viruses, such as herpesviruses, is inactivated in pangolins. Notably, a recent viral metagenomics study demonstrated that, besides RNA viruses, herpesviruses were abundant in pangolins [33]. Given that cGAS is the main cytoplasmic DNA sensor in non-myeloid cells, whereas AIM2 is predominantly expressed in myeloid cells [5], it is possible that epithelial target cells of viruses are particularly compromised in their antiviral response in pangolins. cGAS-STING and IFIH1/MDA5-dependent signaling pathways converge in the production of interferons, and both pathways as well as an interferon of epithelia, IFN- $\varepsilon$ $[26,34]$ are lost in pangolins. Thus, an interdependent evolutionary degeneration of these pathways in pangolins is conceivable.

Importantly, cGAS and STING are not only active in antiviral defense but also in the response to mislocalization of DNA during organelle damage or defective mitosis [3, 5]. cGAS induces phosphorylation of IRF3 and stimulates apoptosis when mitosis is aberrantly arrested [3]. STING is implicated in the control of apoptosis and other modes of cell death in diverse scenarios of cell damage $[5,6]$. Therefore, the absence of cGAS and STING suggests that, besides antiviral defense, the induction of programmed cell death in response to endogenous DNA is reduced in pangolins. It is also interesting to note that ZBP1, which is implicated in the response to endogenous nucleic acids [35-37], is also inactive in pangolins [24].

In contrast to the cGAS and STING pathway, the AIM2dependent response to cytoplasmic DNA appears to be intact in pangolins. Previous studies have shown independent inactivation of AIM2 in several lineages of mammals, including the sister group of pangolins, i.e. carnivorans [18-20]. Other studies from our lab showed peculiar changes in the structure of pro-inflammatory caspases in the cat and the dog [38, 39], indicating that several components of the AIM2 pathway are altered in carnivorans. Finally, it is important to note 
that AIM2 is not functionally equivalent to cGAS (Fig. 3b), suggesting that loss of one of the two pathway cannot be fully compensated by the evolutionary retention of the other.

Taken together, the detection of inactivating mutations in multiple DNA or RNA-sensing proteins indicates a broad, though not complete, degeneration of the response to aberrant localization of exogenous and endogenous nucleic acids in pangolins. The role of pangolins as reservoirs of pathogens with the potential to cause zoonotic spillover and their possible use in comparative studies of immune defense and programmed cell death represent highly interesting fields for future research.

Acknowledgements The research was supported in part by the Austria Science Fund (FWF): P32777. Open access funding provided by Medical University of Vienna.

Author contributions HF, ET, and LE designed the study. HF and LE performed bioinformatic analyses. HF, ET, and LE wrote the manuscript.

Funding The research was supported in part by the Austria Science Fund (FWF): P32777. Open access funding provided by Medical University of Vienna.

Data availability All data and material of this study are publicly available.

\section{Compliance with ethical standards}

Conflict of interest The authors declare that they have no conflict of interest.

Ethical approval Not applicable. No procedures requiring ethics approval were performed in this study.

Open Access This article is licensed under a Creative Commons Attribution 4.0 International License, which permits use, sharing, adaptation, distribution and reproduction in any medium or format, as long as you give appropriate credit to the original author(s) and the source, provide a link to the Creative Commons licence, and indicate if changes were made. The images or other third party material in this article are included in the article's Creative Commons licence, unless indicated otherwise in a credit line to the material. If material is not included in the article's Creative Commons licence and your intended use is not permitted by statutory regulation or exceeds the permitted use, you will need to obtain permission directly from the copyright holder. To view a copy of this licence, visit http://creativecommons.org/licenses/by/4.0/.

\section{References}

1. Ma Z, Ni G, Damania B (2018) Innate sensing of DNA virus genomes. Annu Rev Virol 5:341-362

2. Hu MM, Shu HB (2019) Innate immune response to cytoplasmic DNA: Mechanisms and diseases. Annu Rev Immunol 38:79-98. https://doi.org/10.1146/annurev-immunol-070119-115052
3. Zierhut C, Yamaguchi N, Paredes M, Luo JD, Carroll T, Funabiki $\mathrm{H}$ (2019) The cytoplasmic DNA sensor cGAS promotes mitotic cell death. Cell 178:302-315

4. Ablasser A, Chen ZJ (2019) cGAS in action: Expanding roles in immunity and inflammation. Science 363:eaat8657

5. Paludan SR, Reinert LS, Hornung V (2019) DNA-stimulated cell death: implications for host defence, inflammatory diseases and cancer. Nat Rev Immunol 19:141-153

6. Maelfait J, Liverpool L, Rehwinkel J (2020) Nucleic acid sensors and programmed cell death. J Mol Biol 432:552-568

7. Bürckstümmer T, Baumann C, Blüml S, Dixit E, Dürnberger G, Jahn H, Planyavsky M, Bilban M, Colinge J, Bennett KL, SupertiFurga G (2009) An orthogonal proteomic-genomic screen identifies AIM2 as a cytoplasmic DNA sensor for the inflammasome. Nat Immunol 10:266-272

8. Fernandes-Alnemri T, Yu JW, Datta P, Wu J, Alnemri ES (2009) AIM2 activates the inflammasome and cell death in response to cytoplasmic DNA. Nature 458:509-513

9. Hornung V, Ablasser A, Charrel-Dennis M, Bauernfeind F, Horvath G, Caffrey DR, Latz E, Fitzgerald KA (2009) AIM2 recognizes cytosolic dsDNA and forms a caspase-1-activating inflammasome with ASC. Nature 458:514-518

10. Roberts TL, Idris A, Dunn JA, Kelly GM, Burnton CM, Hodgson S, Hardy LL, Garceau V, Sweet MJ, Ross IL, Hume DA, Stacey KJ (2009) HIN-200 proteins regulate caspase activation in response to foreign cytoplasmic DNA. Science 323:1057-1060

11. Ablasser A, Goldeck M, Cavlar T, Deimling T, Witte G, Röhl I, Hopfner KP, Ludwig J, Hornung V (2013) cGAS produces a 2'-5'-linked cyclic dinucleotide second messenger that activates STING. Nature 498:380-384

12. Gao P, Ascano M, Zillinger T, Wang W, Dai P, Serganov AA, Gaffney BL, Shuman S, Jones RA, Deng L, Hartmann G, Barchet W, Tuschl T, Patel DJ (2013) Structure-function analysis of STING activation by $\mathrm{c}\left[\mathrm{G}\left(2^{\prime}, 5^{\prime}\right) \mathrm{pA}\left(3^{\prime}, 5^{\prime}\right) \mathrm{p}\right]$ and targeting by antiviral DMXAA. Cell 154:748-762

13. Sun L, Wu J, Du F, Chen X, Chen ZJ (2013) Cyclic GMP-AMP synthase is a cytosolic DNA sensor that activates the type I interferon pathway. Science 339:786-791

14. Schoggins JW, MacDuff DA, Imanaka N, Gainey MD, Shrestha B, Eitson JL, Mar KB, Richardson RB, Ratushny AV, Litvak V, Dabelic R, Manicassamy B, Aitchison JD, Aderem A, Elliott RM, García-Sastre A, Racaniello V, Snijder EJ, Yokoyama WM, Diamond MS, Virgin HW, Rice CM (2014) Pan-viral specificity of IFN-induced genes reveals new roles for cGAS in innate immunity. Nature 505:691-695

15. Cohen D, Melamed S, Millman A, Shulman G, OppenheimerShaanan Y, Kacen A, Doron S, Amitai G, Sorek R (2019) Cyclic GMP-AMP signalling protects bacteria against viral infection. Nature 574:691-695

16. Wu X, Wu FH, Wang X, Wang L, Siedow JN, Zhang W, Pei ZM (2014) Molecular evolutionary and structural analysis of the cytosolic DNA sensor cGAS and STING. Nucleic Acids Res 42:8243-8257

17. Kranzusch PJ, Wilson SC, Lee AS, Berger JM, Doudna JA, Vance RE (2015) Ancient origin of cGAS-STING reveals mechanism of universal 2',3' cGAMP signaling. Mol Cell 59:891-903

18. Cridland JA, Curley EZ, Wykes MN, Schroder K, Sweet MJ, Roberts TL, Ragan MA, Kassahn KS, Stacey KJ (2012) The mammalian PYHIN gene family: phylogeny, evolution and expression. BMC Evol Biol 12:140

19. Brunette RL, Young JM, Whitley DG, Brodsky IE, Malik HS, Stetson DB (2012) Extensive evolutionary and functional diversity among mammalian AIM2-like receptors. J Exp Med 209:1969-1983 
20. Ahn M, Cui J, Irving AT, Wang LF (2016) Unique loss of the PYHIN gene family in bats amongst mammals: implications for inflammasome sensing. Sci Rep 6:21722

21. Lam TT, Shum MH, Zhu H, Tong Y, Ni X, Liao Y, Wie W, Cheung WY, Li W, Li L, Leung GM, Holmes EC, Hu Y, Guan Y (2020) Identifying SARS-CoV-2 related coronaviruses in Malayan pangolins. Nature. https://doi.org/10.1038/s41586-020-2169-0

22. Zhang T, Wu Q, Zhang Z (2020) Probable pangolin origin of SARS-CoV-2 associated with the COVID-19 outbreak. Curr Biol 30:1346-1351

23. Andersen KG, Rambaut A, Lipkin WI, Holmes EC, Garry RF (2020) The proximal origin of SARS-CoV-2. Nat Med 26:450-452

24. Fischer H, Tschachler E, Eckhart L (2020) Pangolins lack IFIH1/ MDA5, a cytoplasmic RNA sensor that initiates innate immune defense upon coronavirus infection. Front Immunol 11:939

25. Sharma V, Walther F, Hecker N, Stuckas H, Hiller M (2020) Convergent losses of TLR5 suggest altered extracellular flagellin detection in four mammalian lineages. Mol Biol Evol. https://doi. org $/ 10.1093 / \mathrm{molbev} / \mathrm{msaa} 058$

26. Choo SW, Rayko M, Tan TK, Hari R, Komissarov A, Wee WY, Yurchenko AA, Kliver S, Tamazian G, Antunes A, Wilson RK, Warren WC, Koepfli KP, Minx P, Krasheninnikova K, Kotze A, Dalton DL, Vermaak E, Paterson IC, Dobrynin P, Sitam FT, Rovie-Ryan JJ, Johnson WE, Yusoff AM, Luo SJ, Karuppannan KV, Fang G, Zheng D, Gerstein MB, Lipovich L, O'Brien SJ, Wong GJ (2016) Pangolin genomes and the evolution of mammalian scales and immunity. Genome Res 26:1312-1322

27. Altschul SF, Gish W, Miller W, Myers EW, Lipman DJ (1990) Basic local alignment search tool. J Mol Biol 215:403-410

28. Corpet F (1988) Multiple sequence alignment with hierarchical clustering. Nucleic Acids Res 16:10881-10890

29. Hedges SB, Marin J, Suleski M, Paymer M, Kumar S (2015) Tree of life reveals clock-like speciation and diversification. Mol Biol Evol 32:835-845

30. Unterholzner L, Keating SE, Baran M, Horan KA, Jensen SB, Sharma S, Sirois CM, Jin T, Latz E, Xiao TS, Fitzgerald KA, Paludan SR, Bowie AG (2010) IFI16 is an innate immune sensor for intracellular DNA. Nat Immunol 11:997-1004

31. Almine JF, O'Hare CA, Dunphy G, Haga IR, Naik RJ, Atrih A, Connolly DJ, Taylor J, Kelsall IR, Bowie AG, Beard PM, Unterholzner L (2017) IFI16 and cGAS cooperate in the activation of
STING during DNA sensing in human keratinocytes. Nat Commun 8:14392

32. Jønsson KL, Laustsen A, Krapp C, Skipper KA, Thavachelvam K, Hotter D, Egedal JH, Kjolby M, Mohammadi P, Prabakaran T, Sørensen LK, Sun C, Jensen SB, Holm CK, Lebbink RJ, Johannsen M, Nyegaard M, Mikkelsen JG, Kirchhoff F, Paludan SR, Jakobsen MR (2017) IFI16 is required for DNA sensing in human macrophages by promoting production and function of cGAMP. Nat Commun 8:14391

33. Liu P, Chen W, Chen JP (2019) Viral metagenomics revealed Sendai virus and coronavirus infection of Malayan pangolins (Manis javanica). Viruses 11:979

34. Fung KY, Mangan NE, Cumming H, Horvat JC, Mayall JR, Stifter SA, De Weerd N, Roisman LC, Rossjohn J, Robertson SA, Schjenken JE, Parker B, Gargett CE, Nguyen HP, Carr DJ, Hansbro PM, Hertzog PJ (2013) Interferon- $\varepsilon$ protects the female reproductive tract from viral and bacterial infection. Science 339:1088-1092

35. Devos M, Tanghe G, Gilbert B, Dierick E, Verheirstraeten M, Nemegeer J, de Reuver R, Lefebvre S, De Munck J, Rehwinkel J, Vandenabeele P, Declercq W, Maelfait J (2020) Sensing of endogenous nucleic acids by ZBP1 induces keratinocyte necroptosis and skin inflammation. J Exp Med 217:e20191913

36. Jiao H, Wachsmuth L, Kumari S, Schwarzer R, Lin J, Eren RO, Fisher A, Lane R, Young GR, Kassiotis G, Kaiser WJ, Pasparakis M (2020) Z-nucleic-acid sensing triggers ZBP1-dependent necroptosis and inflammation. Nature 580:391-395

37. Eckhart L, Tschachler E (2018) Control of cell death-associated danger signals during cornification prevents autoinflammation of the skin. Exp Dermatol 27:884-891

38. Eckhart L, Ballaun C, Hermann M, VandeBerg JL, Sipos W, Uthman A, Fischer H, Tschachler E (2008) Identification of novel mammalian caspases reveals an important role of gene loss in shaping the human caspase repertoire. Mol Biol Evol 25:831-841

39. Eckhart L, Ballaun C, Uthman A, Gawlas S, Buchberger M, Fischer H, Tschachler E (2009) Duplication of the caspase-12 prodomain and inactivation of NLRC4/IPAF in the dog. Biochem Biophys Res Commun 384:226-230

Publisher's Note Springer Nature remains neutral with regard to jurisdictional claims in published maps and institutional affiliations. 